\title{
Protocolo clínico de avaliação de doentes adultos com dermatite atópica em tratamento com Dupilumab
}

\section{Clinical protocol of evaluation of adult patients with atopic dermatitis treated with Dupilumab}

Data de receção / Received in: 25/02/2019

Data de aceitação / Accepted for publication in: 18/03/2019

Rev Port Imunoalergologia 20I9;27 (I): I35-I45

Cristina Lopes ${ }^{1,2}$, Marta Neto $^{3}$, Frederico Regateiro ${ }^{4,5}$, João Marcelino ${ }^{3}$, Célia Costa ${ }^{3}$, Sofia Campina ${ }^{6}$, Rita Aguiar ${ }^{6}$, Anabela Lopes ${ }^{3}$; Grupo de Interesse de Alergia Cutânea da Sociedade Portuguesa de Alergologia e Imunologia Clínica

I Unidade de Imunoalergologia Hospital Pedro Hispano Unidade Local de Saúde Matosinhos

${ }^{2}$ Serviço de Imunologia Básica e Clínica, Departamento de Patologia Faculdade de Medicina da Universidade do Porto

${ }^{3}$ Serviço de Imunoalergologia, Hospital Santa Maria, Centro Hospitalar Universitário Lisboa Norte

${ }^{4}$ Serviço de Imunoalergologia, Centro Hospitalar de Coimbra

${ }^{5}$ Instituto de Imunologia, Faculdade de Medicina, Universidade de Coimbra

${ }^{6}$ Unidade de Imunoalergologia do Centro Hospitalar Lisboa Ocidental

\section{RESUMO}

A dermatite atópica (DA) é a patologia cutânea inflamatória mais frequente nos países desenvolvidos sendo frequentemente a primeira manifestação de doença alérgica. Surge habitualmente nos dois primeiros anos de vida, sendo que quando persiste na idade adulta tende a ser mais grave. A sua fisiopatologia é complexa envolvendo factores genéticos e ambientais. $O$ tratamento das formas mais graves inclui a utilização de terapêutica imunossupressora sistémica, sendo o único tratamento on label na Europa nesta categoria, a ciclosporina. O Dupilumab é um anticorpo monoclonal totalmente humano que inibe a sinalização IL-4/IL-13 estando indicado nas formas de dermatite atópica mais graves. Este protocolo clínico pretende estabelecer de acordo com as guidelines internacionais, quais as recomendações clinicas para a utilização de dupilumab em doentes adultos com DA moderada a grave, quais as contraindicações e precauções especiais. Sugere ainda uma proposta de avaliação e monitorização da resposta ao tratamento.

Palavras-chave: Anticorpo monoclonal, dermatite atópica, dupilumab, protocolo, tratamento. 


\section{ABSTRACT}

Atopic dermatitis is the most frequent inflammatory skin disease in developed countries and is often the first manifestation of allergic diseases. It usually appears until two years of age and when persisting into adulthood tends to be more severe. Its physiopathology is complex involving genetic and environmental factors. The treatment of severe forms is based on systemic immunosuppression and cyclosporine is the only drug of this class on label in Europe. Dupilumab is a monoclonal antibody anti-IL-4 IL-I3 indicated in the most severe forms of disease. This clinical protocol intends to establish clinical recommendations based on international guidelines for the use of dupilumab in adult patients with moderate to severe athopic dermatitis. It also approaches contraindications and special precautions proposing a schedule to monitorize treatment response.

Keywords: Atopic dermatitis, dupilumab, monoclonal antibody, protocol, treatment.

\section{DEFINIÇÕES E CONCEITOS GERAIS}

A dermatite atópica (DA) é a patologia cutânea inflamatória mais frequente nos países desenvolvidos'. Os únicos dados disponíveis sobre a prevalência desta patologia em Portugal foram obtidos num estudo de prevalência de DA a nível mundial nas faixas etárias dos 6-7 anos (em Portugal 9,3\%) e 13-14 anos $(5,5 \%)^{2}$. Há, no entanto, a perceção de que a persistência desta patologia até à idade adulta tem vindo a aumentar ${ }^{3}$, assim como o número de novos casos nesta faixa etária ${ }^{4,5}$.

A dermatite atópica caracteriza-se por lesões cutâneas pápulo-vesiculares intensamente pruriginosas que evoluem para descamação, despigmentação e posterior liquenificação. Em cerca de $60-80 \%$ dos casos, a DA surge nos dois primeiros anos de vida, com maior propensão pelo sexo feminino. Na maioria das vezes tem uma evolução favorável, sendo que quando persiste na idade adulta tende a ser mais grave. É frequentemente a primeira manifestação de doença alérgica, sendo que os doentes com DA têm uma incidência aumentada de alergia alimentar, rinite e asma alérgica ${ }^{4,6}$.

A sua fisiopatologia é complexa, envolvendo fatores genéticos (por exemplo, mutações do gene da filagrina) e ambientais (por exemplo, alergénios, alterações do microbioma cutâneo, maior predisposição para colonização por S. aureus). Tem por base uma disfunção da imunidade inata e da imunidade adaptativa com inflamação predominantemente Th2, caracterizada por sobreexpressão de várias citocinas, entre as quais as interleucinas IL-4 e IL-I3, que desempenham funções centrais na fisiopatologia da doença.

O tratamento da DA deve ser orientado de acordo com a sua gravidade, tendo por base a utilização profilática diária de emolientes e a utilização de corticoides e/ou imunossupressores tópicos nas zonas de lesões ativas. No caso das exacerbações agudas pode ser necessária a utilização de corticoides orais por curtos períodos de tempo. Nos casos em que não há remissão dos sintomas com medicação tópica, é geralmente necessária a utilização de terapêutica imunossupressora sistémica, sendo que o único tratamento on label na Europa nesta categoria é a ciclosporina. O metotrexato e a azatioprina têm sido utilizados off label na Europa como alternativas à ciclosporina ${ }^{7}$.

O Dupilumab é um anticorpo monoclonal totalmente humano que tem como alvo a subunidade alfa do recetor da IL-4, desta forma inibindo a sinalização IL-4/IL-138,9. O Dupilumab foi aprovado como tratamento para DA moderada a grave em doentes a partir dos 12 anos pela Food and Drug Administration em março de 2017, na Europa para adultos em setembro de $2017^{\prime \prime}$. Os ensaios clínicos demonstraram uma melhoria significativa quer dos scores 
de gravidade quer da qualidade de vida dos doentes com DA moderada a grave ${ }^{8,9}$.

\section{RECOMENDAÇÕES CLÍNICAS PARA TRATAMENTO COM DUPILUMAB}

O Dupilumab, de acordo com as recomendações internacionais ${ }^{7,10}$, está indicado no tratamento de doentes adultos com DA moderada a grave:

- se não houver resposta a, pelo menos, uma terapêutica sistémica, como ciclosporina (on label), metotrexato, azatioprina e micofenolato de mofetil (off label);

- se estas terapêuticas estiverem contraindicadas ou não tiverem sido toleradas

Na Europa, o único imunomodulador sistémico on label no tratamento da DA é a ciclosporina (dose inicial de 2,5-5 mg/kg/dia, sendo a dose máxima diária de $5 \mathrm{mg} /$ $/ \mathrm{kg} / \mathrm{dia})^{7}$; o tempo expectável para existir resposta ao tratamento é de cerca de 3-6 meses. O tratamento contínuo com ciclosporina não deverá exceder os dois anos ${ }^{7}$.

Recomenda-se que:

- no caso de dúvidas no diagnóstico deve ser ponderada a realização de biópsia cutânea antes de iniciar Dupilumab;

- deve ser ponderada a possibilidade de dermatite de contacto alérgica como fator agravante antes do início de tratamento com Dupilumab.

Relativamente à resposta ao tratamento:

- é expectável observar-se melhoria clínica com Dupilumab a partir das 4 semanas de tratamento;

- deve ser ponderada a suspensão do tratamento com Dupilumab na ausência de resposta adequada às 16 semanas.
Considera-se uma resposta adequada ao tratamento a redução de, pelo menos, $50 \%$ do score EASI (Eczema Area Severity Index) quando comparado com a avaliação inicial e redução de, pelo menos, 4 pontos no score DLQI (Dermatology Life Quality Index) quando comparado com o score inicial.

\section{CONTRAINDICAÇÕES}

Hipersensibilidade à substância ativa ou a qualquer dos excipientes.

Está contraindicada a administração de vacinas com vírus vivos em doentes a fazer tratamento com Dupilumab" . Os doentes a receber Dupilumab podem receber vacinações concomitantes com vacinas inativas ou não vivas ${ }^{\prime \prime}$.

\section{PRECAUÇÕES ESPECIAIS}

\section{Gravidez e lactação}

Não se recomenda a utilização de Dupilumab durante a gravidez ou lactação por não haver dados de segurança durante estes períodos.

\section{Doenças parasitárias ativas}

O Dupilumab pode influenciar a resposta imune contra as infeções helmínticas ao inibir a sinalização IL-4/IL-I3. Se houver elevada suspeição clínica de infeção parasitária, poderá estar indicada a realização de exame parasitológico de fezes. Os doentes com infeções helmínticas preexistentes devem ser tratados antes de iniciarem o Dupilumab. Se os doentes contraírem a infeção enquanto estão a receber tratamento com Dupilumab e não responderem ao tratamento anti-helmíntico, o tratamento com Dupilumab deve ser descontinuado até resolução da infeção.

\section{Conjuntivite}

As conjuntivites são mais comuns nos doentes com DA do que na população em geral - 31 a $56 \%$ dos doen- 
tes com DA desenvolvem conjuntivite, sendo que a prevalência aumenta com a gravidade da $\mathrm{DA}^{\prime}$. Nos ensaios clínicos realizados com Dupilumab durante 52 semanas verificou-se um aumento da incidência anual de episódios de conjuntivite não infeciosa, 9 eventos/ $/ 00$ doentes/ano no grupo placebo para $16-25$ eventos / 100 nos grupos que receberam Dupilumab. ${ }^{9} \mathrm{~A}$ conjuntivite foi mais frequente em doentes com DA mais grave de base, com maior duração da doença e com história clínica prévia de conjuntivite. A maioria dos casos ocorreu no início do tratamento (até aos 4 meses) e foram ligeiros a moderados. Para o tratamento da conjuntivite associada à DA recomenda-se como manutenção a utilização de lágrimas artificiais, sendo que a utilização de fluorometolona $0,1 \%$ colírio e tacrolimus $0,03 \%$ pomada oftálmica demonstraram um benefício claro, com resolução dos sinais e sintomas na maioria dos casos mais graves ${ }^{12}$.

Os doentes que apresentem conjuntivite não infeciosa de novo ou agravamento significativo das queixas prévias de conjuntivite devem ser submetidos a exame oftalmológico.

\section{Asma como comorbilidade}

O Dupilumab obteve aprovação para o tratamento da asma moderada a grave na Food Drug Administration (FDA) em novembro de $2018^{13}$. Os doentes com DA e asma como comorbilidade poderão ter um benefício adicional com o tratamento com Dupilumab.

\section{Imunoterapia com aeroalergénios}

Não existem dados sobre a influência do tratamento com Dupilumab em doentes a fazer tratamento com imunoterapia com alergénios.

\section{Switch de medicação com imunossupressores sistémicos para Dupilumab}

a) Ciclosporina: durante $\circ 1^{\circ}$ mês de tratamento com Dupilumab recomenda-se manter o tratamento com ciclosporina com diminuição progressiva para metade da dose, de 15 em 15 dias até suspensão, de modo a evitar o efeito rebound da suspensão abrupta da ciclosporina;

b) Metotrexato: sem necessidade de período de overlap dada maior semivida do fármaco.

\section{Doentes idosos ( $\geq 65$ anos)}

Não é recomendado qualquer ajuste posológico para os doentes idosos"

\section{Doença renal}

Não é necessário qualquer ajuste posológico nos doentes com insuficiência renal ligeira ou moderada. Estão disponíveis dados muito limitados sobre doentes com insuficiência renal grave"

\section{Doença hepática}

Não estão disponíveis dados sobre doentes com insuficiência hepática"l.

\section{Peso corporal}

Não é recomendado qualquer ajuste posológico para o peso corporal" .

\section{Doentes pediátricos}

A segurança e eficácia do Dupilumab em crianças com menos de 18 anos de idade não foram estabelecidas" .

\section{REGISTOS}

Os doentes candidatos a tratamento com Dupilumab deverão estar registados no Registo de Doentes com dermatite atópica moderada a grave da Sociedade Portuguesa de Alergologia e Imunologia Clinica.

\section{LOCAL E ESQUEMA DE ADMINISTRAÇÃO}

A dose recomendada de Dupilumab para doentes adultos é uma dose inicial de $600 \mathrm{mg}$ (duas injeções de $300 \mathrm{mg}$ ), seguida por $\mathbf{3 0 0 ~ m g ~ a d m i n i s t r a d o s ~ e m ~ s e m a n a s ~ a l t e r n a d a s ~}$ sob a forma de injeção subcutânea na coxa ou no abdómen, 
Quadro I. Esquema de avaliação e monitorização do tratamento com Dupilumab

\begin{tabular}{|c|c|c|c|c|c|c|c|c|c|c|c|}
\hline \multirow{2}{*}{$\begin{array}{l}\text { Parâmetros clínicos } \\
\text { e laboratoriais }\end{array}$} & \multicolumn{11}{|c|}{ Duração do tratamento: semanas(S), meses (M) } \\
\hline & -54 & $\begin{array}{c}\mathrm{s} 0 \\
600 \mathrm{mg}\end{array}$ & $\begin{array}{c}\mathrm{s} 2 \\
300 \mathrm{mg}\end{array}$ & $\begin{array}{l}\text { S4 } \\
\text { MI }\end{array}$ & $\begin{array}{l}\text { S8 } \\
M 2\end{array}$ & $\begin{array}{l}\mathrm{S} 12 \\
\mathrm{M} 3\end{array}$ & $\begin{array}{l}\text { S16 } \\
\text { M4 }\end{array}$ & $\begin{array}{l}\text { S20 } \\
\text { M5 }\end{array}$ & $\begin{array}{l}S 24 \\
\text { M6 }\end{array}$ & $\begin{array}{l}\text { S36 } \\
\text { M9 }\end{array}$ & $\begin{array}{l}S 48 \\
M I 2\end{array}$ \\
\hline Avaliação médica & $\checkmark$ & $\checkmark$ & $\checkmark$ & $\checkmark$ & $\checkmark$ & $\checkmark$ & $\checkmark$ & $\checkmark$ & $\checkmark$ & $\checkmark$ & $\checkmark$ \\
\hline SCORAD & $\checkmark$ & $\checkmark$ & $\checkmark$ & $\checkmark$ & $\checkmark$ & $\checkmark$ & $\checkmark$ & $\checkmark$ & $\checkmark$ & $\checkmark$ & $\checkmark$ \\
\hline EASI & $\checkmark$ & $\checkmark$ & $\checkmark$ & $\checkmark$ & $\checkmark$ & $\checkmark$ & $\checkmark$ & $\checkmark$ & $\checkmark$ & $\checkmark$ & $\checkmark$ \\
\hline DLQI & $\checkmark$ & $\checkmark$ & $\checkmark$ & $\checkmark$ & $\checkmark$ & $\checkmark$ & $\checkmark$ & $\checkmark$ & $\checkmark$ & $\checkmark$ & $\checkmark$ \\
\hline $\begin{array}{l}\beta-H C G \text { (idade fértil) } \\
\text { Hemograma,VS } \\
\lg \text {, IgG, IgA, IgM } \\
\text { Ureia, creatinina } \\
\text { TGO,TGP, Bil. total e direta } \\
\text { Eletroforese sérica }\end{array}$ & $\checkmark$ & & & & & & & & $\checkmark$ & & \\
\hline Registo efeitos adversos & $\checkmark$ & $\checkmark$ & $\checkmark$ & $\checkmark$ & $\checkmark$ & $\checkmark$ & $\checkmark$ & $\checkmark$ & $\checkmark$ & $\checkmark$ & $\checkmark$ \\
\hline Av. oftalmológica & & \multicolumn{10}{|c|}{ Ponderar avaliação se sintomas de conjuntivite não infeciosa em qualquer fase do tratamento } \\
\hline
\end{tabular}

Quadro 2. Esquema de monitorização em doente com diagnóstico de rinite e/ou asma alérgica concomitante

\begin{tabular}{|c|c|c|c|c|c|c|c|c|c|c|c|}
\hline \multirow{2}{*}{$\begin{array}{c}\text { Parâmetros clínicos e } \\
\text { laboratoriais }\end{array}$} & \multicolumn{11}{|c|}{ Semanas de tratamento (S) } \\
\hline & - S4 & $\begin{array}{c}\mathrm{S0} \\
600 \mathrm{mg}\end{array}$ & $\begin{array}{c}S 2 \\
300\end{array}$ & $\begin{array}{l}\text { S4 } \\
\text { MI }\end{array}$ & $\begin{array}{l}\text { S8 } \\
\mathrm{M} 2\end{array}$ & $\begin{array}{l}S 12 \\
M 3\end{array}$ & $\begin{array}{l}S 16 \\
\text { M4 }\end{array}$ & $\begin{array}{l}\text { S20 } \\
\text { M5 }\end{array}$ & $\begin{array}{l}\text { S24 } \\
\text { M6 }\end{array}$ & $\begin{array}{l}\text { S36 } \\
\text { M9 }\end{array}$ & $\begin{array}{l}S 48 \\
M I 2\end{array}$ \\
\hline Questionário CARAT & $\checkmark$ & $\checkmark$ & & $\checkmark$ & & $\checkmark$ & & & $\checkmark$ & & $\checkmark$ \\
\hline $\begin{array}{l}\text { Registo medicação anti- } \\
\text { asmática }\end{array}$ & $\checkmark$ & $\checkmark$ & $\checkmark$ & $\checkmark$ & $\checkmark$ & $\checkmark$ & $\checkmark$ & $\checkmark$ & $\checkmark$ & $\checkmark$ & $\checkmark$ \\
\hline Provas função respiratória & $\checkmark$ & & & & & & & & $\checkmark$ & & $\checkmark$ \\
\hline$N^{\circ}$ exacerbações & \multicolumn{11}{|c|}{$\begin{array}{l}\text { Registo do número de exacerbações com necessidade de corticoterapia oral sistémica } \\
\text { por um período de pelo menos } 3 \text { dias }\end{array}$} \\
\hline
\end{tabular}

excetuando a zona de $5 \mathrm{~cm}$ em redor do umbigo. Se for outra pessoa a administrar, pode ser usada a região superior do braço. Não deve ser administrado em pele lesionada.

Os doentes podem autoinjetar Dupilumab caso o seu profissional de saúde determine que tal é apropriado. Deve ser proporcionada formação adequada aos doentes e/ou aos prestadores de cuidados sobre a preparação e a administração antes da sua utilização.

\section{AVALIAÇÃo}

A avaliação descrita em RCM preconiza apenas uma avaliação médica inicial e às 16 semanas sem necessidade de um controlo analítico específico" . No entanto, sugere-se, para um maior controlo da evolução da resposta ao tratamento, que os doentes com DA a fazer Dupilumab sejam avaliados clinicamente pelo seu médico assistente 
previamente a cada toma no primeiro mês, depois mensalmente até ao $6 .^{\circ}$ mês, depois de $3 / 3$ meses até perfazer um ano e, posteriormente, de $6 / 6$ meses (de acordo com a duração do tratamento).

\section{Gravidade e atividade da doença}

SCORAD (Scoring atopic dermatitis) - utiliza três domínios (extensão, intensidade das lesões e sintomas subjetivos de prurido e perturbação do sono) variando entre 0-103: 0-25-ligeira, 25-50, moderada, grave> 50 (Anexo I).

Eczema Area and Severity Index (EASI) - avalia a extensão e gravidade das lesões $0,1-I, 0=$ muito ligeiro; I, I-7.0 $=$ ligeiro; $7, I-2 I, 0=$ moderado; $2 \mathrm{I}, \mathrm{I}-50,0=$ grave; $50, I-72,0$ $=$ muito grave $($ Anexo 2$)$.

\section{Qualidade de vida}

DLQI-Dermatological life quality of life índex-Questionário validado na população portuguesa com score que varia entre 0 e 30 - sem impacto na qualidade de vida (score 0-I), impacto ligeiro (2-5), moderado (6-10), elevado (II-20), muito elevado (2I-30) ${ }^{14}$ (Anexo 3).

Propõe-se a realização de análises gerais nomeadamente $\beta$-HCG (idade fértil), hemograma, velocidade de sedimentação, doseamento serico de $\lg \mathrm{E}, \lg \mathrm{g}, \lg \mathrm{A}$, IgM, ureia, creatinina TGO, TGP, bilirrubina total e direta, electroforese sérica antes e após 6 meses de tratamento, seguida de uma avaliação anual (Quadro I).

No caso de o doente ter asma como comorbilidade sugere-se a avaliação de controlo de sintomas de rinite e asma através do questionário (CARAT) (disponível em www.new.caratnetwork.org), assim como a realização de provas, função respiratória e monitorização do número de exacerbações de asma (Quadro 2).

\section{EFEITOS INDESEJÁVEIS}

\section{Resumo do perfil de segurança}

As reações adversas mais frequentes foram reações no local de injeção, conjuntivite, blefarite e herpes oral. As reações de hipersensibilidade, incluindo urticária ge- neralizada, exantema, anafilaxia ou doença soro-like, foram reportadas em $<1 \%$ doentes. No RCM não está preconizado que a administração obrigue a vigilância médica.

\section{Sintomas adversos documentados}

- Muito frequentes ( $\geq 1 / 10)$ : Reações no local de administração (inchaço, eritema, dor, prurido);

- Frequentes $(\geq 1 / 100,<1 / 10)$ : conjuntivite, prurido, herpes oral, eosinofilia, cefaleias;

- Muito raros (< I/10.000): doença do soro/reações do tipo doença do soro.

\section{Conflito de interesses}

Os autores não receberam qualquer apoio financeiro para a realização deste protocolo.

Cristina Lopes declara ter recebido apoio financeiro como palestrante ou consultor científico de AstraZeneca, Novartis, TEVA, Sanofi; Frederico Regateiro de AstraZeneca, Novartis, TEVA, Sanofi; Anabela Lopes de Novartis, Sanofi, Bial; Sofia Campina de Novartis; Marta Neto, João Marcelino, Célia Costa e Rita Aguiar não têm conflitos de interesse a declarar.

\section{Contacto:}

Cristina Lopes

E-mail: cristina.lopes.abreu@gmail.com

\section{REFERÊNCIAS}

I. Wollenberg A, Barbarot S, Bieber T, Christen-Zaech S, Deleuran $M$, Fink-Wagner $A$, et al. Consensus-based European guidelines for treatment of atopic eczema (atopic dermatitis) in adults and children: part I. J Eur Acad Dermatol Venereol. 2018;32(5):657-82

2. Odhiambo JA, Williams HC, Clayton TO, Robertson CF, Asher MI, Group IPTS. Global variations in prevalence of eczema symptoms in children from ISAAC phase three. J Allergy Clin Immunol 2009;124:125I-8 el223.

3. Weidinger S, Novak N. Atopic dermatitis. Lancet 2016;387: 1109-22.

4. Lee HH, Patel KR, Singam V, Rastogi S, Silverberg Jl. A systematic review and meta-analysis of the prevalence and phenotype of adult-onset atopic dermatitis. J Am Acad Dermatol 2019;80:1526-32-5 
5. Silverberg JI. Public Health Burden and Epidemiology of Atopic Dermatitis. Dermatol Clin 2017;35:283-9.

6. Weidinger SI NN. Atopic dermatitis revisited. Allergy 2014;69:1-2.

7. Wollenberg A, Barbarot S, Bieber T, Christen-Zaech S, Deleuran $M$, Fink-Wagner A, et al. Consensus-based European guidelines for treatment of atopic eczema (atopic dermatitis) in adults and children: part II. J Eur Acad Dermatol Venereol 2018;32:85078.

8. Thomson J, Wernham AGH, Williams HC. Long-term management of moderate-to-severe atopic dermatitis with Dupilumab and concomitant topical corticosteroids (LIBERTY AD CHRONOS): a critical appraisal. Br J Dermatol 2018;178:897-902.

9. Blauvelt A, de Bruin-Weller M, Gooderham M, Cather JC, Weisman J, Pariser D, et al. Long-term management of moderate-to-severe atopic dermatitis with Dupilumab and concomitant topical corticosteroids (LIBERTY AD CHRONOS): a I-year, randomised, double-blinded, placebo-controlled, phase 3 trial. Lancet 2017;389:2287-303.

10. 2018. Dupilumab for treating moderate to severe atopic dermatitis (TA534). 2018. http://nice.org.uk/guidance/ta534.

II. Resumo Caracteristicas do Medicamento Dupixent $®$ https:// ec.europa.eu/health/documents/communityregister/2017/20170926138667/anx_138667_pt.pdf

12. Wollenberg A, Ariens L, Thurau S, van Luijk C, Seegraber M, de Bruin-Weller M. Conjunctivitis occurring in atopic dermatitis patients treated with Dupilumab-clinical characteristics and treatment. J Allergy Clin Immunol Pract 2018;6:1778-80 .

13 https://www.accessdata.fda.gov/drugsatfda_docs/label/20I7/761055lbl.pdf

14. Finlay AY KG. Dermatology Life Quality Index (DLQI): A simple practical measure for routine clinical use. Clin Exp Dermatol 1994;19:210-6. 
Cristina Lopes, Marta Neto, Frederico Regateiro, João Marcelino, Célia Costa,

Sofia Campina, Rita Aguiar, Anabela Lopes

ANEXO I. ÍNDICE SCORAD (SCORING ATOPIC DERMATITIS)²

A: Extensão da área envolvida: somatório dos valores das áreas envolvidas (áreas entre parêntesis para crianças $<2$ anos)

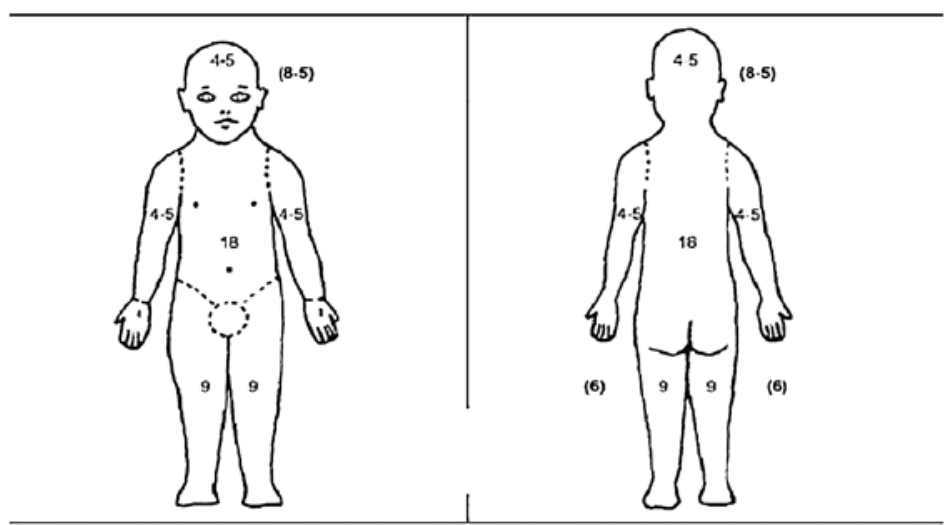

B: Intensidade

\begin{tabular}{|l|l|}
\hline \multicolumn{1}{|c|}{ Critérios } & $\begin{array}{c}\text { Intensidade } \\
(\mathbf{0} \text {-ausente I ligeiro 2= moderado 3 =grave })\end{array}$ \\
\hline Eritema & \\
\hline Edema & \\
\hline Exsudação/crosta & \\
\hline Liquenificação & \\
\hline Secura* & \\
\hline Total & \\
\hline
\end{tabular}

* em áreas de pele não envolvida

C: Sintomas subjetivos (prurido e perturbação do sono) - média dos últimos 3 dias e noites

Prurido

Perturbação sono

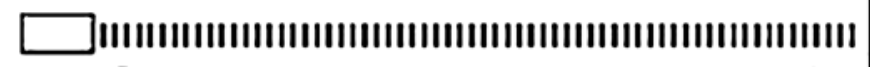

0

JIIIIIIIIIIIIIIIIIIIIIIIIIIIIIIIIIIIIIIIIIIIIIIIIIIII)

SCORAD $=A / 5+7 B / 2+C$ 
ANEXO 2. ECZEMA AREA AND SEVERITY INDEX (EASI) - IDADE > 8 ANOS

Área envolvida: Score 0 a 6 baseado na tabela

\begin{tabular}{|l|l|c|c|c|c|c|c|}
\hline \% Envolvimento & 0 & $1-9 \%$ & $10-29 \%$ & $30-49 \%$ & $50-69 \%$ & $70-89 \%$ & $90-100 \%$ \\
\hline Score de região & 0 & 1 & 2 & 3 & 4 & 5 & 6 \\
\hline
\end{tabular}

Gravidade: Avaliar a gravidade de cada item numa escala de $\mathbf{0}$ a $\mathbf{3}$

\begin{tabular}{|l|l|}
\hline $\mathbf{0}$ & Ausente \\
\hline $\mathbf{I}$ & Ligeira \\
\hline $\mathbf{2}$ & Moderada \\
\hline $\mathbf{3}$ & Grave \\
\hline
\end{tabular}

$\checkmark$ Avaliar o score médio de gravidade na área envolvida

$\checkmark$ Meios pontos (I,5 e 2,5) podem ser usados, não é permitido 0,5

\begin{tabular}{|c|c|c|c|c|c|c|c|}
\hline $\begin{array}{l}\text { Região } \\
\text { corporal }\end{array}$ & $\begin{array}{c}\text { Eritema } \\
(0-3)\end{array}$ & $\begin{array}{c}\text { Edema } \\
(0-3)\end{array}$ & $\begin{array}{c}\text { Escoriação } \\
(0-3)\end{array}$ & $\begin{array}{c}\text { Liquenificaçao } \\
(0-3)\end{array}$ & $\begin{array}{c}\text { Score região } \\
(0-6)\end{array}$ & $\begin{array}{c}\text { Fator } \\
\text { multiplicador }\end{array}$ & $\begin{array}{c}\text { Score } \\
\text { por região } \\
\text { corporal }\end{array}$ \\
\hline $\begin{array}{l}\text { Cabeçal } \\
\text { pescoço }\end{array}$ & & + & + & & $=$ & $\times 0.1$ & \\
\hline Tronco & & + & + & & $=$ & $\times 0.3$ & \\
\hline $\begin{array}{l}\text { Membros } \\
\text { superiores }\end{array}$ & & + & + & & $=$ & $\times 0.2$ & \\
\hline $\begin{array}{l}\text { Membros } \\
\text { inferiores }\end{array}$ & & + & + & & $=$ & $\times 0.4$ & \\
\hline \multicolumn{7}{|c|}{ Score EASI final é a soma dos 4 scores de região: } & $(0-72)$ \\
\hline
\end{tabular}




\section{ÍNDICE DE QUALIDADE DE VIDA - DERMATOLOGIA (IQV-D)}

Forma Experimental Portuguesa, Lisboa, 2004

A preencher pelo(a) médico(a) ou enfermeiro(a):

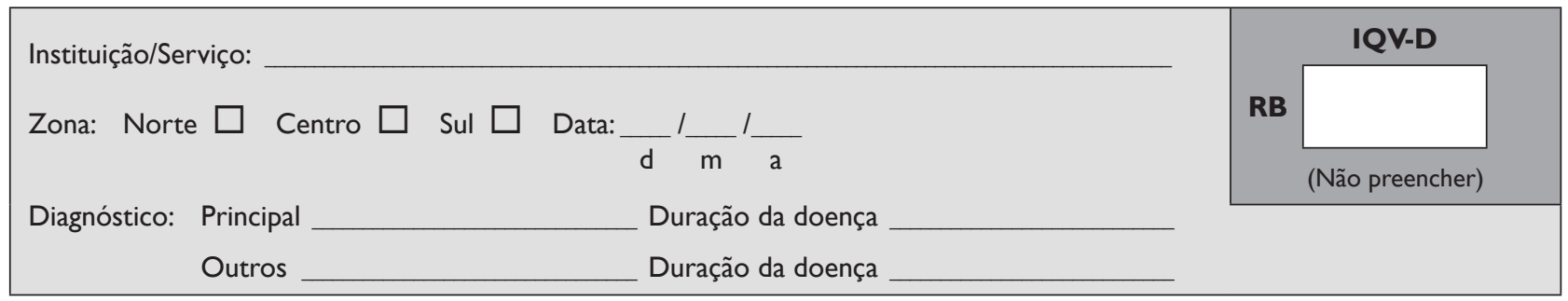

A preencher pelo(a) utente:

\begin{tabular}{llll}
\hline Idade: $\_$Sexo: $\_$Escolaridade: $\_$Profissão: \\
Estado civil: $\quad$ Casado(a)/União de facto $\square \quad$ Separado(a)/Divorciado(a) $\square \quad$ Viúvo(a) $\square \quad$ Solteiro(a) $\square$ \\
\hline
\end{tabular}

\section{QUESTIONÁRIO}

A finalidade do questionário é avaliar quanto o seu problema de pele o(a) afetou DURANTE A ÚLTIMA SEMANA. Por favor marque UMA resposta a cada uma das 10 perguntas.

Assim: $囚$

\begin{tabular}{|c|c|c|c|c|}
\hline I. & $\begin{array}{l}\text { Na última semana sentiu a pele irritada e mais sensível, comichão ou } \\
\text { sensação de picadas? }\end{array}$ & $\begin{array}{l}\text { Muito } \\
\text { Bastante } \\
\text { Um pouco } \\
\text { Nada }\end{array}$ & $\begin{array}{l}\square \\
\square \\
\square \\
\square\end{array}$ & \\
\hline 2. & $\begin{array}{l}\text { Na última semana sentiu-se embaraçado(a) ou incomodado(a) por causa } \\
\text { do estado da sua pele? }\end{array}$ & $\begin{array}{l}\text { Muito } \\
\text { Bastante } \\
\text { Um pouco } \\
\text { Nada }\end{array}$ & $\begin{array}{l}\square \\
\square \\
\square \\
\square\end{array}$ & \\
\hline 3. & $\begin{array}{l}\text { Na última semana até que ponto o seu problema de pele prejudicou a sua } \\
\text { vida normal (fazer compras, cuidar da casa, tratar das plantas, etc.)? }\end{array}$ & $\begin{array}{l}\text { Muito } \\
\text { Bastante } \\
\text { Um pouco } \\
\text { Nada }\end{array}$ & $\begin{array}{l}\square \\
\square \\
\square \\
\square\end{array}$ & Não aplicável $\square$ \\
\hline 4. & $\begin{array}{l}\text { Na última semana a escolha da roupa que usou teve que ver com o estado } \\
\text { da sua pele? }\end{array}$ & $\begin{array}{l}\text { Muito } \\
\text { Bastante } \\
\text { Um pouco } \\
\text { Nada }\end{array}$ & $\begin{array}{l}\square \\
\square \\
\square \\
\square\end{array}$ & Não aplicável $\square$ \\
\hline
\end{tabular}




\begin{tabular}{|c|c|c|c|c|}
\hline 5. & $\begin{array}{l}\text { Na última semana até que ponto o seu problema de pele afetou o convívio } \\
\text { com outras pessoas ou mesmo os seus tempos livres? }\end{array}$ & $\begin{array}{l}\text { Muito } \\
\text { Bastante } \\
\text { Um pouco } \\
\text { Nada }\end{array}$ & $\begin{array}{l}\square \\
\square \\
\square \\
\square\end{array}$ & Não aplicável $\square$ \\
\hline 6. & $\begin{array}{l}\text { Na última semana até que ponto o seu problema de pele o(a) impediu de } \\
\text { praticar desporto? }\end{array}$ & $\begin{array}{l}\text { Muito } \\
\text { Bastante } \\
\text { Um pouco } \\
\text { Nada }\end{array}$ & $\begin{array}{l}\square \\
\square \\
\square \\
\square\end{array}$ & Não aplicável $\square$ \\
\hline 7. & $\begin{array}{l}\text { Na última semana o seu problema de pele impediu-o(a) de trabalhar ou } \\
\text { estudar? }\end{array}$ & $\begin{array}{l}\text { Sim } \\
\text { Não }\end{array}$ & $\square$ & Não aplicável $\square$ \\
\hline & $\begin{array}{l}\text { Se a sua resposta foi } \underline{\mathrm{NA} O} \text {, até que ponto a sua pele foi problema no trabalho ou } \\
\text { na escola? }\end{array}$ & $\begin{array}{l}\text { Bastante } \\
\text { Um pouco } \\
\text { Nada }\end{array}$ & $\square$ & \\
\hline 8. & $\begin{array}{l}\mathrm{Na} \text { última semana o estado da sua pele criou-lhe problemas no } \\
\text { relacionamento com colegas de trabalho, o/a seu/sua companheiro(a), } \\
\text { alguns amigos próximos ou familiares? }\end{array}$ & $\begin{array}{l}\text { Muito } \\
\text { Bastante } \\
\text { Um pouco } \\
\text { Nada }\end{array}$ & $\begin{array}{l}\square \\
\square \\
\square\end{array}$ & Não aplicável $\square$ \\
\hline 9. & $\begin{array}{l}\text { Na última semana até que ponto o seu problema de pele afetou a sua vida } \\
\text { sexual? }\end{array}$ & $\begin{array}{l}\text { Muito } \\
\text { Bastante } \\
\text { Um pouco } \\
\text { Nada }\end{array}$ & $\begin{array}{l}\square \\
\square \\
\square\end{array}$ & Não aplicável $\square$ \\
\hline 10. & $\begin{array}{l}\text { Na última semana até que ponto os tratamentos para a sua pele lhe } \\
\text { criaram problemas, por exemplo de sujar a casa, ou lhe tomarem demasiado } \\
\text { tempo? }\end{array}$ & $\begin{array}{l}\text { Muito } \\
\text { Bastante } \\
\text { Um pouco } \\
\text { Nada }\end{array}$ & $\begin{array}{l}\square \\
\square \\
\square \\
\square\end{array}$ & Não aplicável $\square$ \\
\hline
\end{tabular}

\section{Por favor verifique se respondeu a TODAS as perguntas, e com UMA única resposta a cada uma.} Obrigado.

\section{Todos os direitos reservados}

(C) A.Y. Finlay, G.K. Khan, 1992.

Interdita a reprodução, ainda que parcial, do presente documento.

Versão portuguesa autorizada (cpsicomt@fpce.ul.pt) 